\title{
Datenschutz in Bayerischen Sicherheitsgesetzen unzureichend
}

Der Bayerische Landesbeauftragte für den Datenschutz, Dr. Thomas Petri, begrüßte bei der Sitzung des Verfassungsausschusses des Bayerischen Landtages am 9.07.2009 die von den Koalitionsfraktionen geplanten Änderungen des Polizeiaufgabengesetzes und des Bayerischen Verfassungsschutzgesetzes. Zugleich mahnte er allerdings weitere notwendige Verbesserungen an.

$\mathrm{Zu}$ den seit Jahren vom Landesbeauftragten geforderten Verbesserungen gehört, dass die akustische Überwachung von Privatwohnungen künftig nicht mehr nur automatisiert erfolgen soll. Dadurch wird ermöglicht, dass persönliche Gespräche ohne Ermittlungsrelevanz nicht aufgezeichnet werden. Auch soll auf heimliche Wohnungsdurchsuchungen zur Vorbereitung von Online-Durchsuchungen verzichtet werden. Darüber hinaus soll die verfassungsrechtliche Verpflichtung der Polizei umgesetzt werden, die von der „polizeilichen Beobachtung" Betroffenen zu benachrichtigen. Hiermit wird die gerichtliche Kontrolle der Rechtmäßigkeit dieser Maßnahme erst ermöglicht.

„Nach wie vor sehe ich allerdings nicht, wozu der Verfassungsschutz, der für die Abwehr konkreter Gefahren nicht zuständig ist, die Online-Durchsuchung überhaupt benötigt“, kommentierte Dr. Petri den Entwurf. Diese Überwachungsmaßnahme darf nach den verfassungsrechtlichen Vorgaben nur zur Abwehr konkreter Gefahren eingesetzt werden. Unabhängig davon birgt die derzeitige Doppelzuständigkeit von Polizei und Verfassungsschutz für die Online-Durchsuchung die Gefahr vermehrter Grundrechtseingriffe. Auch ist der Schutz des Kernbereichs privater Lebensgestaltung bei heimlichen Eingriffen des Verfassungsschutzes unzureichend. Das gilt insbesondere für Gespräche mit Berufsgeheimnisträgern, die ohne sachlichen Grund unterschiedlich geschützt werden. Beispielsweise genießen Drogenberater einen höheren Schutz, während Schwangerschaftsberatungsstellen im Vergleich dazu schlechter gestellt werden. Auch insoweit müssen Polizeiaufgabenge- setz und Bayerisches Verfassungsschutzgesetz noch erheblich verbessert werden.

Als Resümee stellt Dr. Petri fest: „Die Änderungen sind bereits wichtige Schritte zur Verbesserung des Grundrechtsschutzes. Es sind aber weitere Schritte dringend notwendig."

\section{Anmerkung der Redaktion:}

Die Bayerischen Sicherheitsgesetze waren bereits im Jahr 2008 Gegenstand dreier Pressemitteilungen des Landesbeauftragten.

Die Mitteilungen vom 17.04. und 2.06.2008 wandte sich gegen einen Gesetzesentwurf, der für Strafverfolgungsbehörden über die Online-Durchsuchung und -überwachung hinaus auch das heimliche Betreten und Durchsuchung von Wohnungen erlauben sollte. Die Mitteilung wies auf erhebliche verfassungsrechtliche Bedenken im Hinblick auf das Grundrecht der Unverletzlichkeit der Wohnung aus Art. 13 GG hin, die nur offene Wohnungsdurchsuchungen erlaube; heimliche Durchsuchungen setzten eine Änderung des Grundgesetzes voraus.

Mit Erklärung vom 6.5.2008 wandte sich der Landesbeauftragte gegen Änderungen des Polizeiaufgabengesetzes, des Bayerischen Verfassungsschutzgesetzes sowie den Entwurf eines Bayerischen Versammlungsgesetzes. Nach der Mitteilung war zwar das Anliegen begrüßenswert, im Polizeiaufgabengesetz die Befugnis zur präventiven Rasterfahndung an die Vorgaben des Bundesverfassungsgerichts anzupassen. Der diesbezügliche Regelungsentwurf enthalte aber keine klaren Regelungen des Zeitpunkts, zu dem die regelmäßig massenhaft erhobenen personenbezogenen Daten gelöscht werden müssten. Außerdem fehle eine verfassungsrechtlich gebotene Benachrichtigungspflicht bei heimlichen Beobachtungen der Polizei. Des Weiteren sei die Befugnis zur automatisierten Kennzeichenerkennung an die Vorgaben des Bundesverfassungsgerichts vom 11.3.2008 anzupassen. Insbesondere sei die Durchführung anlassloser automatisierter Kennzeichenerhebungen und -abgleiche und die Verwendung dieser Daten für die Verfolgung von Ordnungswidrigkeiten und die Abwehr allgemeiner Gefahren für unzulässig. Der Entwurf sehe erstmals für das Landesamt für Verfassungsschutz die Befugnis zur „Online-Durchsuchung“ vor. Das Bundesverfassungsgericht habe eine solche Maßnahme jedoch erst bei Vorliegen tatsächlicher Anhaltspunkte für eine „konkrete Gefahr“ für überragend wichtige Rechtsgüter für zulässig erachtet. Die Abwehr konkreter Gefahren sei aber grundsätzlich Aufgabe der Polizei und der „klassischen“ Sicherheitsbehörden, zu denen das Landesamt für Verfassungsschutz nicht zähle. Das Landesamt für Verfassungsschutz habe als „Frühwarnsystem“ der Staatsregierung dagegen die Aufgabe, im Vorfeld konkreter Gefahren Entwicklungen und Bestrebungen zu beobachten. Eine Befugnis des Landesamts für Verfassungsschutz zur Abwehr konkreter Gefahren sei deshalb systemwidrig. Da auch die bayerische Polizei zu „Online-Durchsuchungen“ ermächtigt werden solle, sei zu befürchten, dass eine solche parallele $\mathrm{Zu}$ ständigkeit von Verfassungsschutz und Polizei ohne ausreichende Abgrenzung zu überlappenden und damit zusätzlichen Rechtseingriffen führe. Der Entwurf eines Bayerischen Versammlungsgesetzes regle erstmals sog. Übersichtsaufzeichnungen und ihre zeitlich unbefristete Speicherung und Nutzung. Bei solchen Aufzeichnungen bestehe die Gefahr, dass auch Bilder personenbezogen erhoben oder durch technische Mittel nachträglich individualisiert werden könnten. Das Bundesverfassungsgericht befürchte, dass potentielle Versammlungsteilnehmer auf eine Teilnahme gerade deshalb verzichten, weil sie nicht abschätzen könnten, ob personenbezogene Informationen dauerhaft gespeichert werden und ihnen daraus Risiken entstehen können. Im Hinblick darauf sollten sog. Übersichtsaufzeichnungen im Gesetz ausdrücklich als „nicht personenbezogene Aufnahmen" definiert und möglichst kurze Löschungsfristen festgelegt werden. Besser wäre allerdings, auf solche Aufzeichnungen völlig zu verzichten. 